\title{
Determination of Moisture Content in 5- Fluorouracil using Diffuse Reflectance Infrared Spectroscopy
}

\author{
Parul Singh, Deepak Kumar Jangir, Ranjana Mehrotra and H.C. Kandpal \\ Optical Radiation Standards, National Physical Laboratory, New Delhi 110 012, India.
}

\begin{abstract}
Determination of moisture content in pharmaceuticals is very important, as moisture is mainly responsible for the degradation of drugs. The degraded drug has not only reduced efficacy but is also hazardous for health. The objective of the present work is to replace the Karl Fischer (KF) titration method used for moisture analysis with a method that is rapid, involves no toxic materials and is more effective. Diffuse reflectance infrared spectroscopy, which is explored as a potential alternate for various applications, is investigated for moisture analysis in 5-Fluorouracil, an anticancer drug.
\end{abstract}

Keywords: Moisture Content; Infrared Spectroscopy; Diffuse Reflectance; 5-Fluorouracil PACS: $01.30 . \mathrm{Cc}$

\section{INTRODUCTION}

5- Fluorouracil is one of the oldest chemotherapy drugs, used as an active medicine against several types of cancers. The present work describes the use of infrared diffuse reflectance spectroscopy for the determination of moisture content in 5-fluorouracil. Determination of moisture content in pharmaceuticals is very important, as moisture is mainly responsible for the degradation of drugs. Moisture content is conventionally determined by thermogravimetric method, Karl Fischer (KF) titration and gas chromatography. These conventional methods are time consuming, expensive and require hazardous chemical reagents. In contrast of these techniques, infrared spectroscopy is advantageous by being rapid, nondestructive and does not require any hazardous chemical reagents.

Infrared spectroscopy has been used by several workers for different applications in pharmaceuticals. It ranges from identification of raw materials $[1,2]$ to moisture determination [3] and process monitoring [4]. The objective of the present work is to evaluate the potential of diffuse reflectance infrared spectroscopy for the determination of moisture content in 5-Fluorouracil. Reference moisture values were obtained by KF titration. A number of calibration models were developed with partial least squares (PLS) regression method. The best calibration model is selected on the bases of minimum root mean square error of calibration (RMSEC) and maximum correlation coefficient $\left(\mathrm{R}^{2}\right)$. 


\section{EXPERIMENTAL}

5- Fluorouracil was kindly supplied by Biochem Pharmaceutical Industries Limited, Daman, India as a gift sample. Specially dried methanol for KF titration was procured from Qualigens Fine Chemicals. Pyridine free $526 \mathrm{KF}$ reagent obtained from Qualigens fine Chemicals was used for KF titration. Potassium bromide (KBr) used was of spectroscopy grade procured from BDH Laboratory Suppliers, England.

A total of one hundred and fifty samples of different moisture content were prepared by exposing the samples to different relative humidity for different timings. Samples were well homogenized before the experiments.

Infrared spectra of the samples were collected in diffuse reflectance mode with Bio $\mathrm{Rad} 175 \mathrm{C}$ Fourier transform infrared spectrophotometer. The spectra with a resolution of $4 \mathrm{~cm}^{-1}$ were obtained by averaging 64 scans. Background spectra were obtained with $\mathrm{KBr}$ powder. Unscrambler 9.1 software was used for data processing and developing calibration model. Savitzky -Golay second order polynomial was used with 10 data points to obtain first and second order derivative spectra.

Reference moisture analysis was carried out by Karl Fischer Titration using LABINDIA automatic KF titrator. For the quantification of moisture content by Karl Fischer titration an accurately weighed (about $300 \mathrm{mg}$ ) amount of 5-Fluorouracil was added to the neutralized methanol and titrated with KF reagent till the end point was reached. KF titrator automatically displayed the percent moisture content of the sample.

\section{RESULTS AND DISCUSSION}

The overlaid infrared spectra of 5-Fluorouracil with different moisture content are shown in Figure 1. There are significant changes in the spectra of the samples as moisture content varies. The absorbance at certain wavelengths increases with moisture content, which shows that there is a quantitative relationship between absorbance and moisture level.

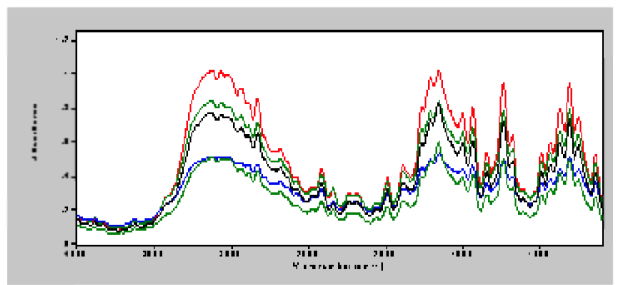

FIGURE 1. Overlaid infrared spectra of 5- Fluorouracil with different moisture content

Calibration was carried out by partial least squares regression method. Different calibration models were developed by combination of several wavelength regions and data pretreatments to determine the wavelength region in which spectral data are best correlated with moisture contents of the samples. The PLS factors were determined for each combination of parameters. The calibration models were developed using KF values as reference for all samples. The optimum number of PLS factors are calculated 
on the bases of minimum root mean square error of prediction (RMSEP). To improve the precision and accuracy of calibration model the outliers, if found, were discarded and the calibration was repeated. The best calibration model was selected on the bases of maximum $\mathrm{R}^{2}$ and minimum RMSEC. Best calibration model is obtained with first derivative spectra in the region of $2600-3600 \mathrm{~cm}^{-1}$. The model has $\mathrm{R}^{2} 0.9751$, RMSEC 0.8615 and no outliers.

A separate set of ten samples was employed for prediction. Spectral measurements were made on these samples and their moisture values are determined using the developed calibration model. KF titration was performed for the same sample set to determine the moisture present. The infrared predicted values were compared with the KF titration values of these samples. Table 1 shows the comparison between IR predicted values and KF titration values. A good correlation is obtained between two values. The results indicate that diffuse reflectance infrared spectroscopy might be used as a rapid, reliable and nondestructive technique for the determination of moisture content in 5-Fluorouracil.

TABLE 1. Actual (KF) Vs Predicted (IR) values of \% moisture in prediction set

\begin{tabular}{cccc}
\hline Sample name & KF values & NIR values & Bias \\
\hline Sample1 & 0.225 & 0.240 & 0.015 \\
Sample2 & 0.184 & 0.366 & 0.182 \\
Sample3 & 3.138 & 3.078 & -0.060 \\
Sample4 & 2.554 & 3.013 & 0.459 \\
Sample5 & 3.684 & 3.385 & -0.299 \\
Sample6 & 3.579 & 2.868 & -0.711 \\
Sample7 & 5.814 & 6.025 & 0.211 \\
Sample 8 & 6.312 & 6.373 & 0.061 \\
Sample 9 & 7.324 & 7.414 & 0.090 \\
Sample 10 & 10.451 & 10.513 & 0.062 \\
\hline
\end{tabular}

\section{ACKNOWLEDGMENTS}

The authors thank the Director, National Physical Laboratory, New Delhi, India for giving permission to publish this paper. PS and DKJ are thankful to Indian Council of Medical Research (ICMR) for providing financial support.

\section{REFERENCES}

1. Y. Roggo, C. Roeseler and M. Ulmschneider, J. Pharm. Biomed. Anal. 36, 777-786 (2004).

2. W. L. Yoon, R.D. Jee and A.C. Moffat, Analyst 125, 1817-1822 (2000).

3. R. A. Mattes, R. Schroeder, V. Dhopeshwarker, R. Kowaland and W. Randolph, Pharmaceutical Tech. Europe, 41-45 (2005).

4. O. R. Dumitrescu, D. C. Baker. G. M. Foster and K. E. Evans, Polymer Testing 24, 367-375 (2005). 